
\title{
25 Research Soure \\ Genome-based taxonomic reclassification of Acinetobacter species using type and reference strains
}

Masato Suzuki ( $\square$ suzuki-m@nih.go.jp )

National Institute of Infectious Diseases https://orcid.org/0000-0001-8975-2193

Shotaro Maehana

Kitasato University

Hidero Kitasato

Kitasato University

\section{Short Report}

Keywords: Acinetobacter, genome-based taxonomic classification, core genome phylogeny, in silico DNA-DNA hybridization, average nucleotide identity

Posted Date: July 6th, 2021

DOl: https://doi.org/10.21203/rs.3.rs-677553/v1

License: (c) (i) This work is licensed under a Creative Commons Attribution 4.0 International License. Read Full License 


\section{Abstract}

Acinetobacter species are widely distributed in the environment and clinical settings worldwide and serve as natural reservoirs of antimicrobial resistance genes and occasional human pathogens responsible for nosocomial infections. In this study, we performed genomic analysis of Acinetobacter seohaensis DSM 16313, a type strain of the proposed Acinetobacter species. This species was estimated to be evolutionary close to Acinetobacter towneri but the genome sequence of $A$. seohaensis was not publicly available. Pangenome analysis of the genome sequence of $A$. seohaensis along with those of genomeavailable type and reference strains of 82 Acinetobacter species including $A$. towneri suggested that three groups of Acinetobacter species, A. seohaensis and A. towneri, Acinetobacter pullorum and Acinetobacter portensis, and Acinetobacter idrijaensis, Acinetobacter mesopotamicus, and Acinetobacter /woffii, were phylogenetically very similar to each other. Genome comparisons based on in silico DNA-DNA hybridization and the average nucleotide identity confirmed that these three groups of Acinetobacter species are conspecific. Based on the rules of priority, A. seohaensis, A. pullorum, and A. idrijaensis/A. mesopotamicus should be reclassified as later heterotypic synonyms of $A$. towneri, $A$. portensis, and $A$. Iwoffii, respectively.

\section{Main Text}

Acinetobacter towneri, belonging to the genus Acinetobacter whose members are gram-negative aerobic coccobacilli, is often isolated from water environments worldwide (1). This species has become increasingly important in recent years as a natural reservoir of antimicrobial resistance (AMR) genes (26). Acinetobacter baumannii, a cause of opportunistic infections in humans, has acquired resistance mechanisms to various antimicrobials, including clinically important carbapenems, making antimicrobial therapy difficult (7). AMR genes, such as those for carbapenem-hydrolyzing enzymes (carbapenemases), have spread among environmental and clinical Acinetobacter species via mobile gene elements such as plasmids (2-4). Tigecycline is a last-resort antimicrobial with promising activity against carbapenemaseproducing gram-negative bacteria, including Acinetobacter species; however, mobile genes for tigecyclineinactivating enzymes, tet $(\mathrm{X})$, have also emerged in $A$. towneri $(5,6)$ and $A$. baumannii $(8-10)$. Accumulation of such clinically relevant AMR genes in environmental bacteria such as $A$. towneri and their transmission to human pathogenic bacteria such as $A$. baumannii poses a global public health threat.

As of July 1, 2021, the List of Prokaryotic names with Standing in Nomenclature (LSPN) listed 92 species of the genus Acinetobacter (https://Ipsn.dsmz.de/genus/acinetobacter). Of these, Acinetobacter venetianus and Acinetobacter refrigeratoris (formerly Acinetobacter refrigeratorensis) were listed in duplicate. Additionally, Acinetobacter grimontii, Acinetobacter guangdongensis, Acinetobacter pakistanensis, and Acinetobacter dijkshoorniae were later identified as different species (Acinetobacter junii, Acinetobacter indicus, Acinetobacter bohemicus, and Acinetobacter lactucae, respectively) (11-14); therefore, the LSPN lists 86 unique species of Acinetobacter (Table S1). Of these, 68 species were validly published in the International Journal of Systematic and Evolutionary Microbiology (IJSEM), whereas the 
remaining 18 species were not validly published (Table S1). To date, the genome sequences of type and reference strains of 79 species are available in the NCBI database, with all 68 species published in the IJSEM (68/68, 100\%) and 11 species not validly published (11/18, 61.1\%) (Table S1). Although not listed in the LSPN, one novel species of Acinetobacter, Acinetobacter kanungonis, has been validly published in the IJSEM (15), and two novel species of Acinetobacter, Acinetobacter rongchengensis and Acinetobacter tianfuensis, have been proposed from large-scale reanalysis on 3,956 genomes of Acinetobacter species in public databases and published in another journal (16) (Table S1).

During molecular epidemiological analysis of carbapenem-resistant $A$. towneri isolates from hospital sewage in Japan, we performed genomic analysis of Acinetobacter seohaensis DSM 16313, a proposed type strain whose genome sequence was estimated to be similar to that of $A$. towneri but the genome sequence of $A$. seohaensis was not publicly available (17). The 16S rRNA gene sequence of $A$. seohaensis DSM 16313 (accession no. AY633608) (17) was shown to be nearly identical (99.8\%) to that of $A$. towneri DSM $14962^{\top}$ (type strain, accession no. EF611416). The Illumina sequencing library (pairedend, insert size 500-900 bp) was prepared using the Nextera XT DNA Library Prep Kit (Illumina). Wholegenome sequencing using the HiSeq $\mathrm{X}$ system (Illumina) was performed, followed by de novo assembly of Illumina reads using Shovill v1.1.0 (https://github.com/tseemann/shovill) with default parameters. The resulting draft genome sequence of $A$. seohaensis DSM 16313 (accession no. BPEQ00000000) consisted of 298 contigs with a genome size of $2,99 \mathrm{Mbp}$ and GC content of $41.3 \%$.

We further performed pangenome analysis of the draft genome sequence of $A$. seohaensis DSM 16313 along with those of genome-available type and reference strains of the aforementioned 82 species of Acinetobacter, including A. towneri DSM $14962^{\top}$, using Roary v3.13.0 (https://github.com/sangerpathogens/Roary) with the parameter of minimum percentage identity for blastp $=60 \%$. Phylogenetic analysis with their core genome using RAxML v8.2.4 (https://github.com/stamatak/standard-RAxML) with default parameters suggested three groups of Acinetobacterspecies, $A$. seohaensis and $A$. towneri; Acinetobacter pullorum and Acinetobacter portensis, and Acinetobacter idrijaensis, Acinetobacter mesopotamicus and Acinetobacter /woffii, to be phylogenetically very similar to each other (Fig. 1). Of these, two pairs of Acinetobacter species, A. pullorum and A. portensis as well as A. mesopotamicus and $A$. Iwoffii, have been suggested to be phylogenetically identical to each other in journals other than the IJSEM published in May 2021 and January 2021, respectively $(16,18)$. We confirmed that the 16S rRNA gene sequences of $A$. pullorum B301 (proposed type strain, accession no. MN909715) and A. portensis $877^{\top}$ (type strain, accession no. KX870877) are nearly identical (99.7\%), and that those of $A$. idrijaensis MII (proposed type strain, GS19_03400 in accession no. JQCU01000127, A. mesopotamicus GC2 (proposed type strain, accession no. KJ867435), and A. Iwoffii NCTC $5866^{\top}$ (type strain, accession no. AB626125) are nearly identical (99.7\% for $A$. idrijaensis compared with $A$. Iwoffii and $99.7 \%$ for $A$. mesopotamicus compared with $A$. Iwoffii), respectively.

The results of in silico DNA-DNA hybridization (DDH) analysis using the Type Strain Genome Server (https://tygs.dsmz.de/) and average nucleotide identity (ANI) analysis using FastANI v1.3 (https://github.com/ParBLiSS/FastANI) confirmed that A. seohaensis DSM 16313 (accession no. 
BPEQ00000000) and A. towneriDSM $14962^{\top}$ (accession no. JHZH00000000) (17) are conspecific with $77.5 \%$ of DDH and $97.7 \%$ of ANI according to their proposed minimal standards ( $\geq 70 \%$ of DDH or $\geq 95 \%$ of ANI) (19) (Fig. 1). Moreover, our results confirmed that A. pullorum B301 (accession no.

JAAARQ000000000) (20) and A. portensis AC $877^{\top}$ (accession no. LWRV00000000) (21) are conspecific with $82.6 \%$ of DDH and $98.6 \%$ of ANI, and that $A$. idrijaensis MII (accession no. JQCU00000000) (22), $A$. mesopotamicus GC2 (accession no. JAALFF000000000) (23), and A. Iwoffii NCTC 5866 ${ }^{\top}$ (accession no. CAADHN000000000) (24) are conspecific with $76.8 \%$ of DDH and $96.1 \%$ of ANI ( $A$. idrijaensis compared with $A$. Iwoffii) and $68.5 \%$ of DDH and $96.0 \%$ of ANI (A. mesopotamicus compared with A. Iwoffii), respectively (Fig. 1). Thus, comparative genomic analysis demonstrated that the aforementioned three groups of Acinetobacter species are conspecific and suggested that genome-level comparisons are essential for proposing novel bacterial species among highly similar species.

The priority of prokaryotic names is governed by the International Code of Nomenclature of Prokaryotes (25). Rule 23a of the code states that, "In a given position, a species can bear only one correct epithet, that is, the earliest that is in accordance with the Rules of this Code". Rules 23b, 24a, and 24b establish the priority of names based on their dates of valid publication in the IJSEM. In our case, A. towneri was validly published in the IJSEM in July 2003 (1) and A. seohaensis was published in another journal in November 2007 (17); A. portensis was validly published in the IJSEM in August 2020 (21) and $A$. pullorum was published in another journal in April 2020 (20); A. Iwoffii was validly published in the International Journal of Systematic and Evolutionary Bacteriology (predecessor journal of the IJSEM) in April 1986 (24), and A. idrijaensis and A. mesopotamicus were published in other journals in November 2014 and October 2020, respectively $(22,23)$. Based on the rules of priority, $A$. seohaensis, A. pullorum, and $A$. idrijaensis/A. mesopotamicus are later heterotypic synonyms of $A$. towneri, $A$. portensis, and $A$. Iwoffii, respectively.

\section{References}

1. Carr EL, Kämpfer P, Patel BKC, Gürtler V, Seviour RJ. Seven novel species of Acinetobacterisolated from activated sludge. Int J Syst Evol Microbiol. 2003 53(Pt 4):953-963.

2. Zou D, Huang Y, Liu W, Yang Z, Dong D, Huang S, He X, Ao D, Liu N, Wang S, Wang Y, Tong Y, Yuan J, Huang L. Complete sequences of two novel b/a NDM-1 $_{1}$-harbouring plasmids from two Acinetobacter towneri isolates in China associated with the acquisition of Tn 125. Sci Rep. 2017 7(1):9405.

3. Jiang N, Zhang X, Zhou Y, Zhang Z, Zheng X. Whole-genome sequencing of an NDM-1- and OXA-58producing Acinetobacter towneri isolate from hospital sewage in Sichuan Province, China. J Glob Antimicrob Resist. 2019 16:4-5.

4. Wang K, Li P, Li J, Hu X, Lin Y, Yang L, Qiu S, Ma H, Li P, Song H. An NDM-1-Producing Acinetobacter towneri Isolate from Hospital Sewage in China. Infect Drug Resist. 2020 13:1105-1110. 
5. Ma J, Wang J, Feng J, Liu Y, Yang B, Li R, Bai L, He T, Wang X, Yang Z. Characterization of Three Porcine Acinetobacter towneri Strains Co-Harboring tet(X3) and bla $\mathrm{OXA}_{-58}$. Front Cell Infect

Microbiol. 2020 10:586507.

6. Cheng Y, Chen Y, Liu Y, Song J, Chen Y, Shan T, Xiao Y, Zhou K. Detection of a new tet(X6)-encoding plasmid in Acinetobacter towneri. J Glob Antimicrob Resist. 2021 25:132-136.

7. Piperaki ET, Tzouvelekis LS, Miriagou V, Daikos GL. Carbapenem-resistant Acinetobacter baumannir: in pursuit of an effective treatment. Clin Microbiol Infect. 2019 25(8):951-957.

8. He T, Wang R, Liu D, Walsh TR, Zhang R, Lv Y, Ke Y, Ji Q, Wei R, Liu Z, Shen Y, Wang G, Sun L, Lei L, Lv Z, Li Y, Pang M, Wang L, Sun Q, Fu Y, Song H, Hao Y, Shen Z, Wang S, Chen G, Wu C, Shen J, Wang Y. Emergence of plasmid-mediated high-level tigecycline resistance genes in animals and humans. Nat Microbiol. 2019 4(9):1450-1456.

9. Wang L, Liu D, Lv Y, Cui L, Li Y, Li T, Song H, Hao Y, Shen J, Wang Y, Walsh TR. Novel PlasmidMediated tet(X5) Gene Conferring Resistance to Tigecycline, Eravacycline, and Omadacycline in a Clinical Acinetobacter baumannii Isolate. Antimicrob Agents Chemother. 2019 64(1):e01326-19.

10. Liu D, Zhai W, Song H, Fu Y, Schwarz S, He T, Bai L, Wang Y, Walsh TR, Shen J. Identification of the novel tigecycline resistance gene tet(X6) and its variants in Myroides, Acinetobacter and Proteus of food animal origin. J Antimicrob Chemother. 2020 75(6):1428-1431.

11. Vaneechoutte M, De Baere T, Nemec A, Musílek M, van der Reijden TJ, Dijkshoorn L. Reclassification of Acinetobacter grimontii Carr et al. 2003 as a later synonym of Acinetobacter junii Bouvet and Grimont 1986. Int J Syst Evol Microbiol. 2008 58(Pt 4):937-40.

12. Nemec A, Radolfova-Krizova L. Acinetobacter guangdongensis Feng et al. 2014 is a junior heterotypic synonym of Acinetobacter indicus Malhotra et al. 2012. Int J Syst Evol Microbiol. 2017 67(10):40804082.

13. Nemec A, Radolfova-Krizova L. Acinetobacter pakistanensis Abbas et al. 2014 is a later heterotypic synonym of Acinetobacter bohemicus Krizova et al. 2014. Int J Syst Evol Microbiol. 2016 66(12):56145617.

14. Dunlap CA, Rooney AP. Acinetobacter dijkshoorniae is a later heterotypic synonym of Acinetobacter lactucae. Int J Syst Evol Microbiol. 2018 68(1):131-132.

15. Das L, Deb S, Das SK. Description of Acinetobacter kanungonis sp. nov., based on phylogenomic analysis. Int J Syst Evol Microbiol. 2021 71(6).

16. Qin J, Feng Y, Lü X, Zong Z. Precise Species Identification for Acinetobacter. a Genome-Based Study with Description of Two Novel Acinetobacter Species. mSystems. 2021 e0023721. 
17. Yoon JH, Kim IG, Oh TK. Acinetobacter marinus sp. nov. and Acinetobacter seohaensis sp. nov., isolated from sea water of the Yellow Sea in Korea. J Microbiol Biotechnol. 2007 17(11):1743-50.

18. Nemec A. Strain "Acinetobacter mesopotamicus" GC2 Does Not Represent a Novel Species, but Belongs to the Species Acinetobacter Iwoffii as Revealed by Whole-Genome Sequence-Based Analysis. Curr Microbiol. 2021 78(1):369-370.

19. Chun J, Oren A, Ventosa A, Christensen H, Arahal DR, da Costa MS, Rooney AP, Yi H, Xu XW, De Meyer S, Trujillo ME. Proposed minimal standards for the use of genome data for the taxonomy of prokaryotes. Int J Syst Evol Microbiol. 2018 68(1):461-466.

20. Elnar AG, Kim MG, Lee JE, Han RH, Yoon SH, Lee GY, Yang SJ, Kim GB. Acinetobacter pullorum sp. nov., Isolated from Chicken Meat. J Microbiol Biotechnol. 2020 30(4):526-532.

21. Carvalheira A, Gonzales-Siles L, Salvà-Serra F, Lindgren Å, Svensson-Stadler L, Thorell K, PiñeiroIglesias B, Karlsson R, Silva J, Teixeira P, Moore ERB. Acinetobacter portensis sp. nov. and Acinetobacter guerrae sp. nov., isolated from raw meat. Int J Syst Evol Microbiol. 2020 70(8):4544-4554.

22. Campos-Guillén J, Caballero Pérez J, Cruz Medina JA, Molina Vera C, Salas Rosas LM, Limpens Gutiérrez C, García Salinas I, Hernández Ramírez MR, Soto Alonso G, Cruz Hernández A, Saldaña Gutiérrez C, Romero Gómez S, Pastrana Martínez X, Alvarez Hidalgo E, Gosar M, Dizdarevič T. Draft Genome Sequence of the Mercury-Resistant Bacterium Acinetobacter idrijaensis Strain MII, Isolated from a MineImpacted Area, Idrija, Slovenia. Genome Announc. 2014 2(6):e01177-14.

23. Acer Ö, Güven K, Poli A, Di Donato P, Leone L, Buono L, Güven RG, Nicolaus B, Finore I. Acinetobacter mesopotamicus sp. nov., Petroleum-degrading Bacterium, Isolated from Petroleum-Contaminated Soil in Diyarbakir, in the Southeast of Turkey. Curr Microbiol. 2020 77(10):3192-3200.

24. Bouvet PJM, Grimont PAD. Taxonomy of the Genus Acinetobacter with the Recognition of Acinetobacter baumannii sp. nov., Acinetobacter haemolyticus sp. nov., Acinetobacter johnsonii sp. nov., and Acinetobacter junii sp. nov. and Emended Descriptions of Acinetobacter calcoaceticus and Acinetobacter Iwoffii. Int J Syst Evol Bacteriol. 1986 36(2): 228-240

25. Parker CT, Tindall BJ, Garrity GM. International Code of Nomenclature of Prokaryotes Prokaryotic Code (2008 Revision). Int J Syst Evol Microbiol. 2019 69(1A):S1-S111.

\section{Competing Interests}

The authors declare no competing interests.

\section{Figures}



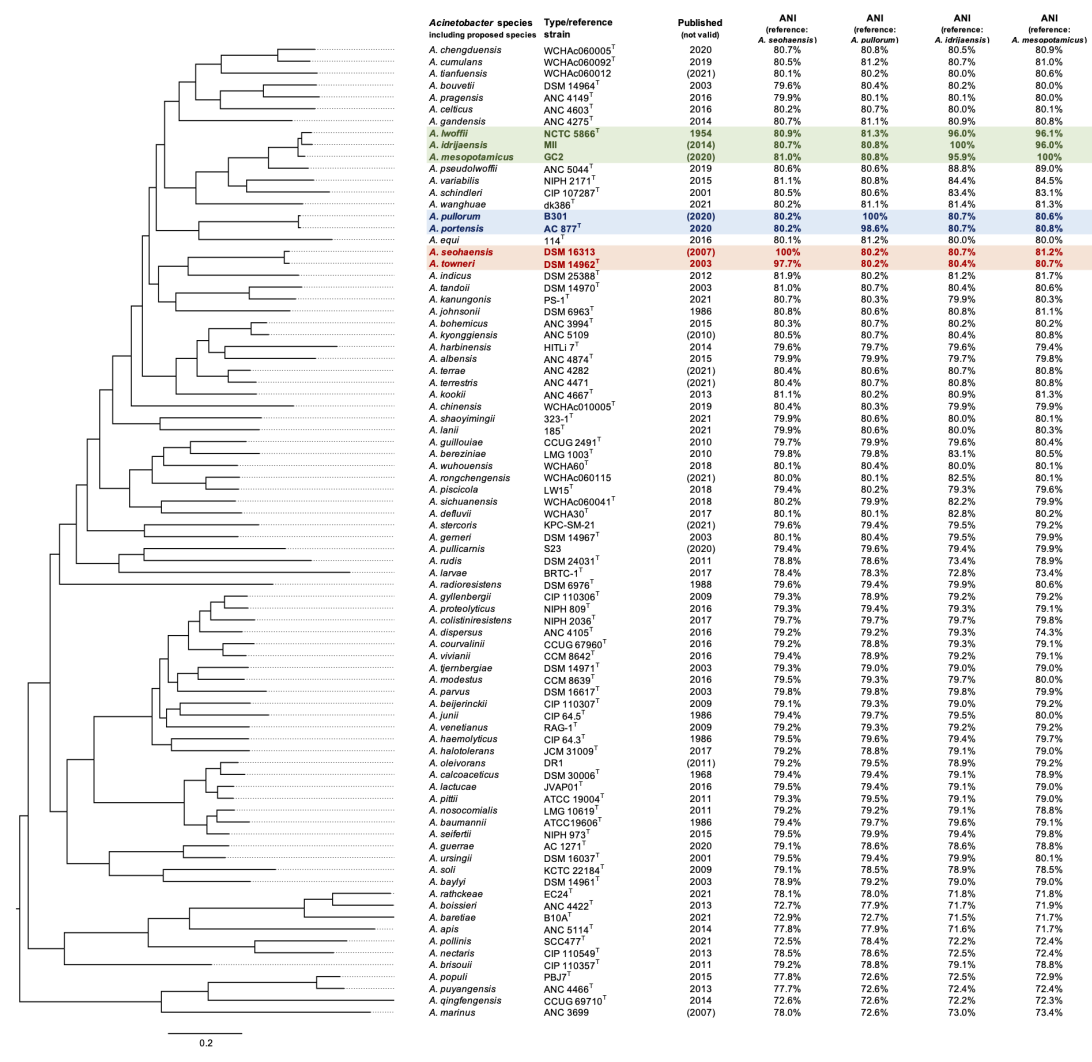

Fig. 1

\section{Figure 1}

Core genome phylogeny of publicly available genomes of type and reference strains of 83 species of Acinetobacter. Bar lengths represent the number of substitutions per site in the core genome. Names of type and reference strains of Acinetobacter species, accession nos. of the genome sequences, years when each strain was validly published in the International Journal of Systematic and Evolutionary Microbiology (or years when each strain was published in another journal), and average nucleotide 
identity (ANI) values to reference genomes of A. seohaensis DSM 16313, A. pullorum B301, A. idrijaensis MII, and A. mesopotamicus GC2, respectively, are shown. Three groups of Acinetobacter species, A. seohaensis and A. towneri; Acinetobacter pullorum and Acinetobacter portensis; and Acinetobacter idrijaensis, Acinetobacter mesopotamicus, and Acinetobacter Iwoffii are highlighted in red, blue, and green, respectively.

\section{Supplementary Files}

This is a list of supplementary files associated with this preprint. Click to download.

- 210626TableS1.pdf 